\title{
Role of Teledentistry in COVID-19 Pandemic: \\ A Nationwide Comparative Analysis among Dental Professionals
}

\author{
Beenish Abbas ${ }^{1}$ Mehreen Wajahat ${ }^{2} \quad$ Zakia Saleem $^{3}$ \\ Zohaib Khurshid ${ }^{6, \odot}$ \\ ${ }^{1}$ Department of Pediatric Dentistry, Foundation University, College \\ of Dentistry, Rawalpindi, Pakistan \\ 2Department of Dental Materials, Avicenna Dental College, \\ Lahore, Pakistan \\ ${ }^{3}$ Department of Dental Education, University College of Dentistry, \\ Lahore, Pakistan \\ ${ }^{4}$ Department of Dental Materials, Dental College, HITEC-Institute of \\ Medical Sciences, Rawalpindi, Pakistan \\ ${ }^{5}$ Department of Dental Materials, Avicenna Dental College, \\ Lahore, Pakistan \\ ${ }^{6}$ Department of Prosthodontics and Dental Implantology, College \\ of Dentistry, King Faisal University, Al Ahsa, Saudi Arabia
}

Eur J Dent:2020;14(suppl S1):S116-S122
Eisha Imran ${ }^{4} \quad$ Mehvish Sajjad ${ }^{5}$

Address for correspondence Mehreen Wajahat, BDS, MPhil, Department of Dental Materials, Avicenna Dental College, Lahore 54810, Pakistan (e-mail: mehrinwajahat09@gmail.com).

\begin{abstract}
Keywords

- teledentistry

- pandemic

- COVID-19

Objectives To assess knowledge, awareness, and practice of health care workers about the role of teledentistry in coronavirus disease 2019 (COVID-19).

Materials and Methods A prevalidated structured questionnaire was used to evaluate awareness of general dentists, postgraduate dental students, dental educators, and consultants with postgraduation degrees toward teledentistry. The acceptability, reliability, and language clarity were also pretested. A total of 510 dental professionals contributed to this study.

Statistical Analysis A statistical analysis using SPSS (version 20.0) was performed. Responses were noted as yes or no. A $p$-value of $\leq 0.05$ was considered to be significant. Frequencies described data. Chi-square test was performed for intergroup comparison to evaluate if the knowledge and attitude diverge with the increase in the level of seniority in the profession.

Results Awareness regarding teledentistry is high among general dentists with bachelor of dental surgery qualification in comparison to final-year students and postgraduate dental students. The majority of participants agreed with improvement in health care $(88.20 \%)$, access to rural areas $(82.90 \%)$, and general practicing dentist agreed with statistically significant difference $(0.00)$ on saving time by teledentistry. Conclusion General practicing dentist was observed with high awareness of teledentistry as compared with postgraduate and undergraduate dental students.
\end{abstract}

DOI https://doi.org/ 10.1055/s-0040-1722107 ISSN 1305-7456. (c) 2020. European Journal of Dentistry.

This is an open access article published by Thieme under the terms of the Creative Commons Attribution-NonDerivative-NonCommercial-License, permitting copying and reproduction so long as the original work is given appropriate credit. Contents may not be used for commercial purposes, or adapted, remixed, transformed or built upon. (https://creativecommons.org/licenses/by-nc-nd/4.0/)

Thieme Medical and Scientific Publishers Pvt. Ltd., A-12, 2nd Floor, Sector 2, Noida-201301 UP, India 


\section{Introduction}

Teledentistry is defined as providing dental care, advice, and treatment remotely by making use of telecommunication technology to avoid direct personal contact with patients. The use of teledentistry is already established within disciplines of oral surgery, oral medicine, periodontal conditions, early detection of caries, and most importantly spreading preventive advice to masses. Telediagnosis, teleconsultation, patient's electronic records, and electronic patient referrals are some of the key modes used in modern day dental practice. ${ }^{1}$ Teledentistry is not limited by time and space can be used to provide interactive access to specialist opinion in a less intimidating way to provide advice about treatment by making the use of telecommunication and information technology over a distance. Photographs, video images, data, history, and records can be shared between participants who are physically separated for a reason. ${ }^{2}$ To reduce the burden on frontliners and to flatten the curve of coronavirus disease 2019 (COVID-19), guidelines have been issued by American Dental Association (ADA). These guidelines state that all nonemergency oral health care facilities should be postponed and only emergency care is to be provided, for example, conditions such as uncontrolled bleeding, cellulitis, and trauma to facial bones that compromises the airway (Teledentistry update: navigating teledentistry during the COVID-19 pandemic (United States). Healthcare Alert, COVID-19 Alert March 27, 2020, by Russell Sass, Adriana Valldejuly, and Summer Galitz). ADA advised dentists worldwide to make use of teledentistry to limit COVID-19 spread and audiovisual telecommunication technology can be used to share live video between patient and health care worker. Health information (e.g., patient's radiographs, photographs, digital impressions, etc.) can be shared through a secure electronic communication system to a health care practitioner. A dentist can deliver advice based on patients' information without real-time live contact. ${ }^{3}$

A protective physical barrier can be created, making use of teledentistry in COVID-19 surge, a screening protocol can be established where practitioner can conduct the audiovisual examination on a stable patient via video conferencing. Implementation of such programs can be an efficient and cost-effective way to ensure the safety of patient and clinician during the pandemic. This strategy can be helpful in lifting strain from health care facilities. ${ }^{4}$ Teledentistry can be useful to tackle the issue of uneven distribution of specialist health care providers. Patient-centered services can be provided to underprivileged communities and oral health-related information can be disseminated effectively using teledentistry. Collaboration and peer-to-peer distant learning is crucial to meet ever increasing knowledge advancements in various disciplines of dentistry and telecommunication technology can be relied upon in this regard. ${ }^{5}$

Recent COVID-19 pandemic has identified the need to use virtual encounter functions that have been underutilized. There is need of time to expand electronic medical record systems and to spread awareness among health care providers to bring teledentistry in practice so as to exercise social distancing while still not compromising patient's care. ${ }^{6}$ Organizations and health care systems worldwide are switching to e-health care technologies as a crucial ally to deal with this pandemic and widen the coverage of health facilities, limiting unnecessary visits realizing the seriousness of situation. Teledentistry makes use of technology to enable healing at a distance while exercising social distancing. Data privacy, adequate training, and access to the internet are essential requirements to engage in this initiative of teledentistry. ${ }^{7}$ Reported benefits of teledentistry are increased access, minimal cost, reduced stress of transportation, and easy access to specialist practice. Most urgent cases that need immediate attention can be tackled using community health care programs. ${ }^{8}$ In the current pandemic, most health care workers are unable to provide sustainable dental care to all patients without risking the spread of disease due to a reduction in workforce and limitation of resources. We propose a paradigm shift to creating a virtual e-dentistry program through the introduction of user-friendly technology to provide an effective foundation for sustainable dental care while prioritizing risk reduction for both patient and dentist. ${ }^{9}$ According to a recent systematic review, teledentistry is a valid tool comparable with face-to-face oral identification of disease, accurate diagnosis, and teleconsultations. Electronic specialist referrals are possible without putting undue strain on frontliners. ${ }^{10}$

Worldwide adoption of virtual dental practices and telephonic consultations will limit overcrowding of emergency services, potentially limiting burden on health care systems. These measures if adopted wisely can prevent exposure of health care workers and patients to COVID-19 and asymptomatic carriers. Services of high-risk staff who are immunocompromised, old, or pregnant can be utilized as they can work remotely. ${ }^{11}$ Live real-time video consultations can be utilized to diagnose intraoral and extraoral soft tissue swelling, mobile teeth, periodontal problems, and grossly carious dentition to make a reliable diagnosis. Image quality, lighting, internet connection, and patient's technology literacy are important factors required to be considered. ${ }^{12}$

\section{Materials and Methods}

This cross-sectional survey was performed among dental professionals to assess the knowledge, awareness, and practice of teledentistry during the COVID-19 pandemic. Dental professionals from all over the country (Pakistan) participated in this digital survey which was designed in English. Those who were unwilling to participate did not take this survey. A prevalidated structured questionnaire was utilized for this purpose..$^{13}$ The acceptability, reliability, and language clarity were pretested. A total of 510 dental professionals contributed to this study. Dental professionals included general dentists, postgraduate dental students, dental educators, and consultants with postgraduation degrees.

Ethical Review Board of University College of Dentistry, University of Lahore reviewed and provided the scientific and ethical approval for carrying out the present research. 
Participants were given plentiful time to fill the questionnaire. This participation on voluntary grounds was considered as informed consent of each participant to this study. Confidentiality of each participant was maintained.

Data were statistically analyzed using SPSS version 20.0. Responses after getting information on the level of seniority were recorded as yes or no. A $p$-value of $\leq 0.05$ was considered to be significant. The data were described in frequencies. Chi-square test was performed for intergroup comparison to evaluate if the knowledge and attitude diverge with the increase in the level of seniority in the profession.

Considering limitations to this study, the researcher stumbled upon the problem in obtaining the required number of responses. Since this study included dental professionals from all across the country, ethical approval from all the institutions was not achievable.

\section{Results}

Qualification was taken as a demographic variable. Percentage of each category was calculated. According to that percentage, participants with bachelor of dental surgery (BDS) as qualification were $57.6 \%$, participants with master of philosophy (MPhil)/membership college of physicians (MCPS) as qualification were $8.2 \%$, participants with the qualification of BDS with postgraduate training continued were $18.8 \%$, participants with fellowship college of physicians and surgeons (FCPS)/master of dental surgery (MDS)/doctor of philosophy (PhD) were $4.1 \%$, and students of BDS final year were $11.2 \%$, as shown in - Fig. 1.

On asking about the familiarity of dentists with the word teledentistry, $72.40 \%$ responded positively and $76.0 \%$ reported that teledentistry could really be a great help in health education. As per $35.90 \%$ dentists' opinion, teledentistry could be applied in every branch of dentistry, whereas the majority of dentists negated this statement (64.10\%). According to 88.20\% of participants, teledentistry could be useful in improving access to oral health care; $75.90 \%$ dentists' opinion about teledentistry as violating patient's privacy was negative; $84.10 \%$ dentists believed that teledentistry is a time-saving

\section{Qualification}

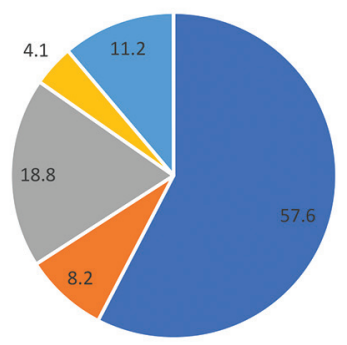

technique; $53.50 \%$ dentists reported that teledentistry helps provide a good understanding of patient's oral health problem over the Internet. As per $67.60 \%$ dentists, teledentistry is a source of reducing specialist's isolation by providing peer contact and specialist report; $52.40 \%$ dentists agreed upon teledentistry as the convenient type of oral health care delivery for making dental examination easier; 62.90\% dentists negated that teledentistry helps monitor patient's condition thoroughly. Teledentistry provides $75.30 \%$ dentists reported the platform of expert consultation; 55.30\% dentists responded negatively regarding the difficulties of Internetrelated equipment usage; $61.20 \%$ dentists said that the risk of data entry mistakes related to teledentistry is unavoidable; $73.50 \%$ dentists reported that teledentistry is a more natural way to contact the patient ( $\mathbf{- T a b l e} \mathbf{1}$ ).

Moreover, to explore the comparison of qualification with perception of teledentistry benefits, chi-square test was applied which showed significant difference. Total 35.9\% dentists (BDS), 7.1\% dentists (MPhil/MCPS), 18.2\% dentists (BDS + postgraduate trainee), 4.1\% dentists (FCPS/MDS/PhD), and $7.1 \%$ dentists (final-year students) were familiar with the term teledentistry (chi-square test $=58.06, p<0.001$ ).

Table 1 Opinion of dentists about teledentistry in response percentage

\begin{tabular}{|l|l|l|}
\hline Statements & Yes & No \\
\hline Have you heard about teledentistry & $72.40 \%$ & $27.60 \%$ \\
\hline $\begin{array}{l}\text { Can teledentistry really help in health } \\
\text { education }\end{array}$ & $76.50 \%$ & $23.50 \%$ \\
\hline $\begin{array}{l}\text { Can teledentistry be applied in every branch } \\
\text { of dentistry }\end{array}$ & $35.90 \%$ & $64.10 \%$ \\
\hline $\begin{array}{l}\text { Can teledentistry be useful in improving } \\
\text { access to oral health care }\end{array}$ & $88.20 \%$ & $11.80 \%$ \\
\hline $\begin{array}{l}\text { Your opinion about teledentistry as violating } \\
\text { patient's privacy }\end{array}$ & $24.10 \%$ & $75.90 \%$ \\
\hline $\begin{array}{l}\text { Does teledentistry increase accessibility } \\
\text { of specialists to rural and underserved } \\
\text { communities for their dental needs }\end{array}$ & $82.90 \%$ & $17.10 \%$ \\
\hline Is teledentistry a time-saving technique & $84.10 \%$ & $15.90 \%$ \\
\hline $\begin{array}{l}\text { Is teledentistry helpful in providing good } \\
\text { understanding of patient's oral health } \\
\text { problem over Internet }\end{array}$ & $53.50 \%$ & $46.50 \%$ \\
\hline $\begin{array}{l}\text { Can teledentistry reduce isolation of } \\
\text { specialist by providing peer contact and } \\
\text { specialist report }\end{array}$ & $67.60 \%$ & $32.40 \%$ \\
\hline $\begin{array}{l}\text { Is teledentistry a convenient type of oral } \\
\text { health care delivery for making dental } \\
\text { examination easier }\end{array}$ & $52.40 \%$ & $47.60 \%$ \\
\hline $\begin{array}{l}\text { Does teledentistry help monitoring } \\
\text { patient's condition well }\end{array}$ & $75.30 \%$ & $24.70 \%$ \\
\hline $\begin{array}{l}\text { Does teledentistry provide platform of } \\
\text { expert consultation }\end{array}$ & $74.70 \%$ & $55.30 \%$ \\
\hline $\begin{array}{l}\text { Do you face difficulties in Internet-related } \\
\text { equipment usage }\end{array}$ & $62.90 \%$ \\
\hline $\begin{array}{l}\text { Is there any risk of data entry mistakes } \\
\text { related to teledentistry }\end{array}$ & $38.80 \%$ \\
\hline $\begin{array}{l}\text { Is it easier to contact patient via } \\
\text { teledentistry }\end{array}$ & $26.50 \%$ \\
\hline
\end{tabular}

Fig. 1 Qualification of participants. BDS, bachelor of dental surgery; FCPS, fellowship college of physicians and surgeons; MCPS, membership college of physicians; MDS, master of dental surgery; MPhil, master of philosophy; PhD, doctor of philosophy.

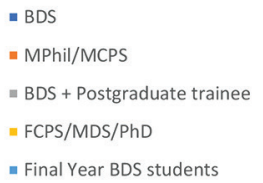

European Journal of Dentistry Vol. 14 No. S1/2020 (c) 2020. European Journal of Dentistry. 
Positive responses were shown by $41.2 \%$ dentists (BDS), $6.5 \%$ dentists (MPhil/MCPS), $16.5 \%$ dentists (BDS + postgraduate trainee), $4.1 \%$ dentists (FCPS/MDS/PhD), and $8.2 \%$ dentists (final-year students) about teledentistry as a supporting technique for health education (chi-square test $=17.45$, $p<0.001$ ). Total 21.2\% (BDS), 2.4\% dentists (MPhil/MCPS), $4.7 \%$ dentists (BDS + postgraduate trainee), 3.5\% dentists (FCPS/MDS/PhD), and $4.1 \%$ dentists (final-year students) supported the statement that teledentistry could be applied in every branch of dentistry (chi-square test $=28.70$, $p<0.001$ ). Positive responses were reported by $51.8 \%$ dentists (BDS), 6.5\% dentists (MPhil/MCPS), 16.5\% dentists (BDS + postgraduate trainee), 4.1\% dentists (FCPS/MDS/PhD), and $9.4 \%$ dentists (final-year students) about teledentistry as a useful technique in improving access to oral health care (chi-square test $=8.21, p=0.08$ ). Total $15.9 \%$ dentists (BDS), $2.4 \%$ dentists (MPhil/MCPS), $2.4 \%$ dentists (BDS + postgraduate trainee), $1.2 \%$ dentists (FCPS/MDS/PhD), and $2.4 \%$ dentists (final-year students) perceived that teledentistry implementation is about violating patient's privacy (chi-square test $=9.94, p<0.04$ ). Total $48.2 \%$ dentists (BDS), $6.5 \%$ dentists (MPhil/MCPS), 15.3\% dentists (BDS + postgraduate trainee), 3.5\% dentists (FCPS/MDS/PhD), and 9.4\% dentists (final-year students) reported that teledentistry increases accessibility of specialists to rural and underserved communities for their dental needs (chi-square test $=1.05, p=0.90$ ). Total $51.8 \%$ dentists (BDS), 6.5\% dentists (MPhil/MCPS), 14.1\% dentists (BDS + postgraduate trainee), 3.5\% dentists (FCPS/MDS/ $\mathrm{PhD}$ ), and $8.2 \%$ dentists (final-year students) reported that teledentistry is a time-saving technique (chi-square test = $18.72, p<0.001$ ). Teledentistry is helpful in providing good understanding of patient's oral health problem over Internet was favored by $31.8 \%$ dentists (BDS), 5.9\% dentists (MPhil/ MCPS), $6.5 \%$ dentists (BDS + postgraduate trainee), 3.5\% dentists (FCPS/MDS/PhD), and $5.9 \%$ dentists (final-year students) (chi-square test $=28.64, p<0.001$ ). According to $39.4 \%$ dentists (BDS), 3.5\% dentists (MPhil/MCPS), 11.8\% dentists (BDS + postgraduate trainee), $4.1 \%$ dentists (FCPS/MDS/PhD), and 8.8\% dentists (final-year students), teledentistry can reduce isolation of specialist by providing peer contact and specialist report (chi-square test $=26.39, p<0.001$ ). According to $31.8 \%$ dentists (BDS), $4.1 \%$ dentists (MPhil/MCPS), $7.1 \%$ dentists (BDS + postgraduate trainee), $2.9 \%$ dentists (FCPS/ $\mathrm{MDS} / \mathrm{PhD}$ ), and $6.5 \%$ dentists (final-year students), teledentistry is a convenient type of oral health care delivery for making dental examination easier (chi-square test $=13.23$, $p<0.01$ ). According to $21.2 \%$ dentists (BDS), $3.5 \%$ dentists (MPhil/MCPS), 6.5\% dentists (BDS + postgraduate trainee), $3.5 \%$ dentists (FCPS/MDS/PhD), and $2.4 \%$ dentists (final-year students), teledentistry helps monitoring patient's condition well (chi-square test $=28.48, p<0.001$ ). According to $43.5 \%$ dentists (BDS), $5.3 \%$ dentists (MPhil/MCPS), $13.5 \%$ dentists (BDS + postgraduate trainee), 3.5\% dentists (FCPS/ $\mathrm{MDS} / \mathrm{PhD}$ ), and $9.4 \%$ dentists (final-year students), teledentistry provides platform of expert consultation (chi-square test $=7.00, p=0.13$ ). According to $27.6 \%$ dentists (BDS), $1.8 \%$ dentists (MPhil/MCPS), $8.2 \%$ dentists (BDS + postgraduate trainee), $0.6 \%$ dentists (FCPS/MDS/PhD), and 6.5\% dentists (final-year students), they face difficulties of Internet-related equipment usage (chi-square test $=22.37, p<0.001$ ). Total 39.4\% dentists (BDS), 2.9\% dentists (MPhil/MCPS), 8.8\% dentists (BDS + postgraduate trainee), $1.8 \%$ dentists (FCPS/MDS/ $\mathrm{PhD}$ ), and $8.2 \%$ dentists (final-year students) showed their concerns about data entry mistakes related to teledentistry (chi-square test $=32.85, p<0.001$ ). Teledentistry makes patient's contact easier was favored by $42.4 \%$ dentists (BDS), $6.5 \%$ dentists (MPhil/MCPS), $12.4 \%$ dentists (BDS + postgraduate trainee), $4.1 \%$ dentists (FCPS/MDS/PhD), and $8.2 \%$ dentists (final-year students) $($ chi-square test $=11.19, p<0.02$ ) (-Table 2).

\section{Discussion}

COVID-19 pandemic has resulted in massive disruptions in the field of health care, while dentistry is considered as one of very risky professions in present circumstances. Aerosols and droplets being the main source of transmission enhance the chance of infection for dental practitioners. A cross-sectional study with participants from around the globe reported the fear and anxiety among dental practitioners due to ongoing pandemic. ${ }^{14-16} \mathrm{~A}$ web-based survey showed negative influence of COVID-19 pandemic on dental clinical routine due to closure of offices. ${ }^{17}$ On the other hand, disruption of patient care cannot be paused as it will result in the increase of dental diseases. It indicates the need to utilize the safest way to ensure the provision of dental care in the pandemic. ${ }^{18}$

Teledentistry in the present time of COVID-19 is the only most reliable way to provide consultation to patients which require communication using technology. Significance of teledentistry has also been highlighted in the literature. Communication for teleconsultation can be made via applications for instant messaging or video calling. Consultation via WhatsApp has been reported as a beneficial option for teledentistry. ${ }^{19}$ Teledentistry is a mean of avoiding person-to-person contact while satisfying the requirement of social distancing by facilitating remote dental care, patient education, and guidance which is advocated by health care authorities across the globe.Teledentistry has various subunits such as teleconsultation, telediagnosis, telemonitoring, and teletriage. Teleconsultation helps in reducing nonurgent patient referrals and reduces burden on busy health care facilities. Telediagnosis makes use of technology to exchange patient records, intraoral images, and radiographic images to diagnose oral pathologies remotely. ${ }^{20}$ Teletriage prioritizes patients requiring urgent dental care by doing remote assessment of oral health reducing the need of unnecessary travel keeping in view socioeconomic and geographical difficulties. ${ }^{21}$ The dental practitioner can have safe access to patients by using the technology for consultation and management of some dental issues..$^{22} \mathrm{~A}$ literature review also indicates useful application of teledentistry in various branches of dentistry that is, field of oral medicine and diagnosis, oral and maxillofacial surgery, endodontics, orthodontics, prosthodontics, periodontics, periodontics, pediatric, and preventive dentistry. ${ }^{23}$

The present study performed in Pakistan was meant to survey and compare the perceptions of general dentist, 
Table 2 Qualification wise difference in terms of teledentistry conceptualization

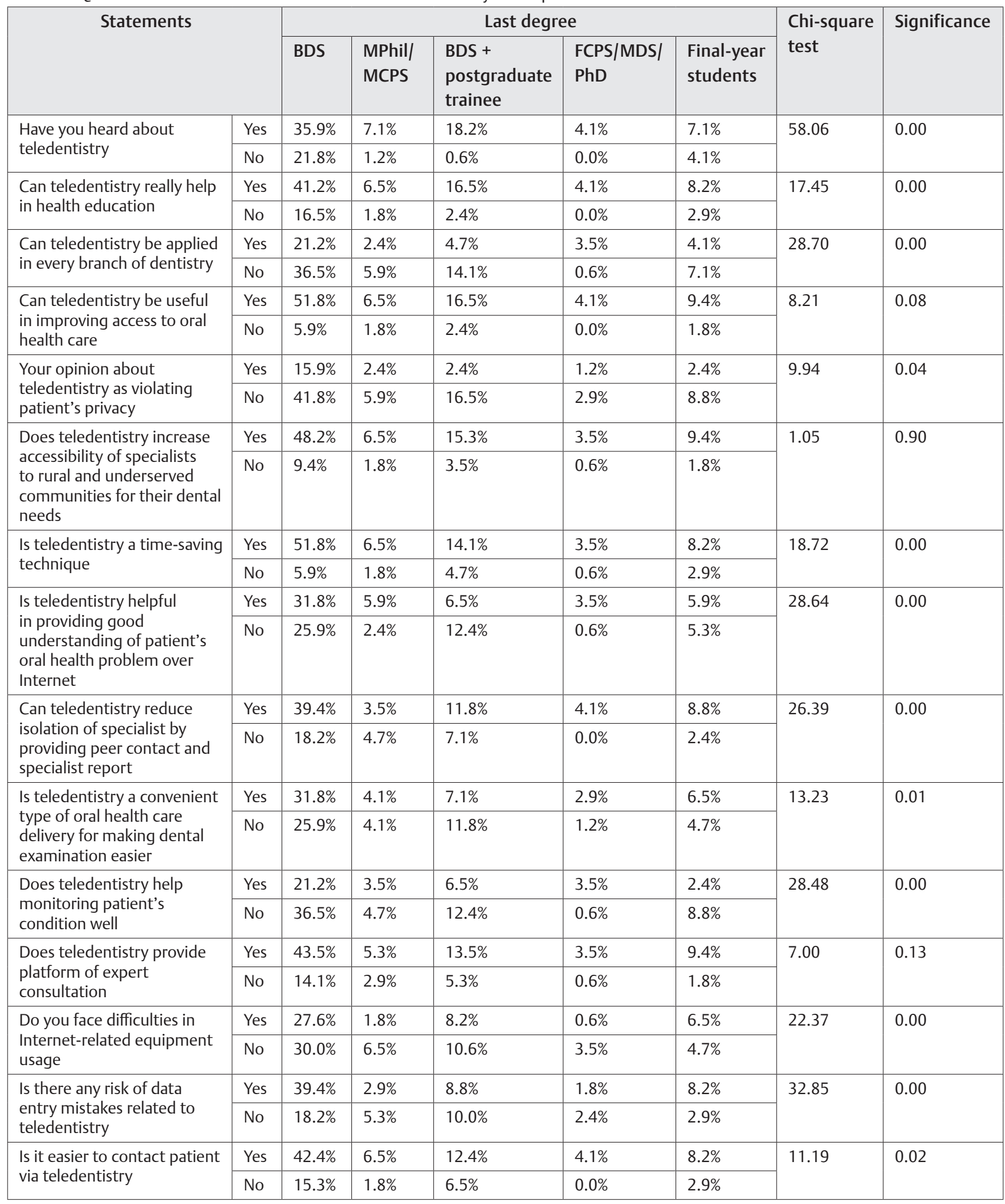

Abbreviations: BDS, bachelor of dental surgery; FCPS, fellowship college of physicians and surgeons; MCPS, membership college of physicians; MDS, master of dental surgery; MPhil, master of philosophy; PhD, doctor of philosophy.

undergraduate and postgraduate dental students regarding teledentistry. The participants (72.40\%) of the present study had knowledge about the "teledentistry." Studies published in other countries, about the perception of teledentistry, also showed similar findings. ${ }^{24}$
Access to rural and distant areas has been reported as one of the main benefits of teledentistry. Before COVID-19 pandemic, teledentistry has been used for patient counseling in distant and rural areas. This trait can be utilized in present condition while observing social distancing from patients. 
A quality improvement study addressing the oral health care in old age residents in the rural areas of Queensland reported improved health care plans with minimization of need to attend an oral health care system with the intervention of teledentistry. ${ }^{25}$ Participants $(82.90 \%)$ of the present study agreed on the provision of better access to rural areas and $88.20 \%$ have a perception of improvement in access to oral health care. A pilot study performed at the regional center of reference for COVID-19 in Italy also states teledentistry as a beneficial tool in the management of patients at a reduced cost while avoiding waiting durations. ${ }^{26}$ Changing demographics, lack of resources, poor access to oral screening, and treatment are a major hindrance to quality care in remote and rural areas. Screening and consultation of patients by means of teledentistry are considered as feasible and accessable. ${ }^{27}$

A study performed in 2015 by Mamatha states that $50 \%$ of the practicing dentist agree on promotion and execution of health education by teledentistry, ${ }^{28}$ which is parallel to the finding of the current study. Health education by teledentistry has been widely used. It is divided into two main categories: self-instruction and video conferencing. Both methods have been used in different countries and mentioned in several studies. ${ }^{29}$

Total $35.9 \%$ of participants of the study believe on its application in every branch of dentistry which is close to a study performed in India where $42 \%$ of dentists were observed with similar perception. ${ }^{30}$ Bradley et al mentioned the benefits and use of teledentistry in the branch of oral medicine. ${ }^{31}$ Telemedicine approach was approved equal to live clinical diagnostic assessment of impacted and semi-impacted molars. ${ }^{32}$ Telecommunication by means of teledentistry was proved useful for diagnosis of periapical lesion in endodontics. ${ }^{33}$ Diagnosis with treatment planning has been performed in prosthodontics by video conferencing. ${ }^{34}$ Along with these, application to pediatric dentistry, periodontics, and orthodontics has been mentioned in the studies. $^{35-37}$

Advancement in technology also impacted the health care and education system, which results in ease of communication between professionals. Dental professionals found it beneficial to discuss patient-related problems with the expert in the respective field and it also helps in establishment of strong networking. ${ }^{38}$ Sixty-seven per cent of the present study participants agree with the fact to promote effective official networking with peers and experts in the field.

According to the present study, awareness regarding teledentistry is high among general dentists with BDS qualification in comparison to final-year students and postgraduate dental students. It is in contrast to a study by Ata and Ozkan, which states less awareness among general dentist in comparison to postgraduate and undergraduate students. ${ }^{39}$ Further research can unveil the reason of high awareness of teledentistry among general practicing dentists.

Dental professional's fast go to teledentistry in the intense period of the COVID-19 pandemic gives an amazing chance to investigate how teledentistry can be embraced in dental care. Teledentistry may prompt a change of standard dental practices and requires authoritative, clinical, innovative, and moral guidelines. Teledentistry features the possibility to offer extra dental care past dental crises, during and post COVID-19. With the fast advancement of correspondence and data innovations and their utilization in our lives, dentistry must change accordingly. ${ }^{40}$

\section{Conclusion}

In the present study, significance of teledentistry has been established and highlighted. General practicing dentists were observed with high awareness of teledentistry. Postgraduate and undergraduate dental students were observed having less awareness. Further research is required for qualitative findings on difference in perceptions among general dentist, undergraduate student, and postgraduate student regarding teledentisty.

\section{Conflict of Interest}

None declared.

\section{References}

1 Kumar N, John N, Devi N, Vivek S, Ravishankar P, Somaraj V. Teledentistry: An overview. J Clin Res Dent 2019;2(2):1-2

2 Bhargava A, Sabbarwal B, Jaggi A, Chand S, Tandon S. Teledentistry: A literature review of evolution and ethicolegal aspects. J Global Oral Health 2019;2(2):128-133

3 Flores APDC, Lazaro SA, Molina-Bastos CG, et al. Teledentistry in the diagnosis of oral lesions: a systematic review of the literature. J Am Med Inform Assoc 2020;27(7):1166-1172

4 Chou E, Hsieh Y-L, Wolfshohl J, Green F, Bhakta T. Onsite telemedicine strategy for coronavirus (COVID-19) screening to limit exposure in ED. Emerg Med J 2020;37(6):335-337

5 Nichols K. Teledentistry overview: United States of America. J Int Soc Telemed eHealth 2019;7:e9

6 Al Kasab S, Almallouhi E, Holmstedt CA. Optimizing the use of teleneurology during the COVID-19 pandemic. Telemed J E Health 2020;26(10):1197-1198

7 Leite H, Hodgkinson IR, Gruber T. New Development: 'Healing at a distance'-Telemedicine and COVID-19. Public Money Manag 2020;40(6):483-485

8 McLaren SW, Kopycka-Kedzierawski DT, Nordfelt J. Accuracy of teledentistry examinations at predicting actual treatment modality in a pediatric dentistry clinic. J Telemed Telecare 2017 ;23(8):710-715

9 Estai M, Bunt SM, Kanagasingam Y, Kruger E, Tennant M. A resource reallocation model for school dental screening: taking advantage of teledentistry in low-risk areas. Int Dent J 2018;68(4):262-268

10 Alabdullah JH, Daniel SJ. A systematic review on the validity of teledentistry. Telemed J E Health 2018;24(8):639-648

11 Rahman N, Nathwani S, Kandiah T. Teledentistry from a patient perspective during the coronavirus pandemic [published online ahead of print, 2020 Aug 14]. Br Dent J 2020;1-4 doi:10.1038/s41415-020-1919-6

12 Patel T, Wong J. The role of real-time interactive video consultations in dental practice during the recovery and restoration phase of the COVID-19 outbreak. Br Dent J 2020 229(3):196-200

13 Verma K, Bhaskar D, Kaur N, Yadav P, Sharma V, Gupta H. Knowledge, attitude and practices regarding teledentistry 
among dental professionals of Mathura city, India. University J Dental Sciences 2019;5(3):49-52

14 Ahmed MA, Jouhar R, Ahmed N, et al. Fear and practice modifications among dentists to combat novel coronavirus disease (COVID-19) outbreak. Int J Environ Res Public Health 2020;17(8):2821

15 Nasir EF, Elhag AK, Almahdi HM. COVID-19 perceptional disparity among dental healthcare personnel at King Faisal University: Applying health belief model (HBM). Eur J Dent 2020;14(suppl S1):S56-S62 doi:10.1055/s-0040-1716782

16 Melo Neto CLM, Bannwart LC, de Melo Moreno AL, Goiato MC. SARS-CoV-2 and dentistry-review. Eur J Dent 2020;14(suppl S1):S130-S139 doi:10.1055/s-0040-1716438

17 Faccini M, Ferruzzi F, Mori AA, et al. Dental care during COVID-19 outbreak: A web-based survey. Eur J Dent 2020; 14(suppl S1):S14-S19 doi:10.1055/s-0040-1715990

18 Coulthard P. Dentistry and coronavirus (COVID-19) - moral decision-making. Br Dent J 2020;228(7):503-505

19 Telles-Araujo GdT, Caminha RD, Kallás MS, Santos PSdS. Teledentistry support in COVID-19 oral care. Clinics 2020;75:e2030

20 Dar-Odeh N, Babkair H, Alnazzawi A, Abu-Hammad S, AbuHammad A, Abu-Hammad O. Utilization of teledentistry in antimicrobial prescribing and diagnosis of infectious diseases during COVID-19 lockdown. Eur J Dent 2020;14(suppl S1): S20-S26 doi:10.1055/s-0040-1717159

21 Ghai S. Teledentistry during COVID-19 pandemic. Diabetes Metab Syndr 2020;14(5):933-935

22 Niazi MIK, Ghafoor S. Teledentistry and COVID-19; today and tomorrow. Biomedica 2020;36(COVID19-S2):81-83

23 Jampani ND, Nutalapati R, Dontula BS, Boyapati R. Applications of teledentistry: a literature review and update. J Int Soc Prev Community Dent 2011;1(2):37-44

24 Al-Khalifa KS, AlSheikh R. Teledentistry awareness among dental professionals in Saudi Arabia. PLoS One 2020; 15(10): 0240825

25 Tynan A, Deeth L, McKenzie D, et al. Integrated approach to oral health in aged care facilities using oral health practitioners and teledentistry in rural Queensland. Aust J Rural Health 2018;26(4):290-294

26 Giudice A, Barone S, Muraca D, et al. Can teledentistry improve the monitoring of patients during the covid-19 dissemination? A descriptive pilot study. Int J Environ Res Public Health 2020;17(10):3399

27 Daniel SJ, Kumar S. Teledentistry: a key component in access to care. J Evid Based Dent Pract 2014;14(Suppl):201-208
28 Boringi M, Waghray S, Lavanya R, et al. Knowledge and awareness of teledentistry among dental professionals-a cross sectional study. J Clin Diagn Res 2015;9(8):ZC41-ZC44

29 Chen J-W, Hobdell MH, Dunn K, Johnson KA, Zhang J. Teledentistry and its use in dental education. J Am Dent Assoc 2003;134(3):342-346

30 Balsaraf SV, Chole RH. Knowledge, awareness, and attitude among practicing dentists about teledentistry in Indore, Central India. J Indian Assoc Public Health Dent 2015;13:434-437

31 Bradley M, Black P, Noble S, Thompson R, Lamey PJ. Application of teledentistry in oral medicine in a community dental service, N. Ireland. Br Dent J 2010;209(8):399-404

32 Duka M, Mihailović B, Miladinović M, Janković A, Vujicić B. [Evaluation of telemedicine systems for impacted third molars diagnosis]. Vojnosanit Pregl 2009;66(12):985-991

33 Živković D, Tošić G, Mihailović B, Miladinović M, Vujičić B. Diagnosis of periapical lesions of the front teeth using the Internet. PONS Medicinski Časopis 2010;7(4):138-143

34 Ignatius E, Perälä S, Mäkelä K. Use of videoconferencing for consultation in dental prosthetics and oral rehabilitation. J Telemed Telecare 2010;16(8):467-470

35 Berndt J, Leone P, King G. Using teledentistry to provide interceptive orthodontic services to disadvantaged children. Am J Orthod Dentofacial Orthop 2008;134(5):700-706

36 Rocca MA, Kudryk VL, Pajak JC, Morris T, eds. The evolution of a teledentistry system within the Department of Defense. Proceedings of the AMIA Symposium; American Medical Informatics Association; 1999

37 Kopycka-Kedzierawski DT, Billings RJ. Prevalence of dental caries and dental care utilisation in preschool urban children enrolled in a comparative-effectiveness study. Eur Arch Paediatr Dent 2011;12(3):133-138

38 Hovliaras CA, Savvy Success: Achieving Professional Excellence and Career Satisfaction in the Dental Hygiene Profession. Vol. II Patient Care. AuthorHouse; 2012

39 Ata SO, Ozkan S, eds. Information technology in oral health care: attitudes of dental professionals on the use of teledentistry in turkey. European and Mediterranean Conference on Information Systems; 2009

40 Talla PK, Levin L, Glogauer M, Cable C, Allison PJ. Delivering dental care as we emerge from the initial phase of the COVID-19 pandemic: teledentistry and face-to-face consultations in a new clinical world. Quintessence Int 2020;51(8): 672-677 